\title{
On the variable effect of mosaic normal/balanced chromosomal rearrangements in man
}

\author{
A Kleczkowska, J P Fryns, H Van den Berghe
}

\begin{abstract}
In this report we summarise the Leuven experience of mosaic normal/balanced chromosomal rearrangements in man, and we review the few published reports on this subject.
\end{abstract}

\section{Material, methods, and results}

In the period 1966 to 1989 we performed a constitutional chromosome analysis in 74306 patients. In 245 index patients a balanced autosomal reciprocal translocation, other than a Robertsonian type translocation, was found. In 75 probands, a paracentric $(29 / 75)$ or a pericentric $(46 / 75)$ inversion was detected. In these two groups of index patients, six were found to have a mosaic normal/balanced chromosomal rearrangement. In all six patients the mosaicism was confirmed in two different peripheral blood lymphocyte cultures ( $G$ and $R$ banding). The percentage of cells with the chromosomal rearrangement was remarkably stable in the different cultures in all patients. Three (two males, one female) had mosaic normal/balanced reciprocal autosomal translocations, and three others (one male, two females) had a mosaic normal/'balanced' inversion. Brief clinical, cytogenetic, and family data of the six patients are shown in the table.

A mosaic normal/reciprocal 1;9 translocation was detected in a 27 year old normal male (patient 1 ). This mosaic normal/reciprocal autosomal translocation was a fortuitous finding as chromosomal analysis was performed in this man and his normal spouse for psychological reasons (spouse working as a social nurse in an institute for the mentally retarded). The first pregnancy ended in a spontaneous abortion. In the second and third pregnancies amniocentesis was performed at 15 weeks' gestation. Chromosome analysis showed a female karyotype with an identical,

Centre for Human Genetics, Herestraat 49, B-3000 Leuven, Belgium.

A Kleczkowska, J P Fryns, H Van den Berghe Correspondence to Professor Fryns.

Received for publication 25 January 1990.

Accepted for publication 2 March 1990. non-mosaic 1;9 translocation in both fetuses. Both children were phenotypically normal at birth and had normal psychomotor development. A mosaic normal/ reciprocal 9;13 translocation was diagnosed in the 29 year old husband of a couple who had had two spontaneous first trimester abortions (patient 2) and a mosaic normal/insertional 13;14 translocation was found in a 24 year old female with trilogy of Fallot (patient 3). She was sent for genetic counselling after the birth of a son with tetralogy of Fallot. Chromosomal analysis in this boy was normal. A mosaic normal/inv(12)(q12q24) (patient 4) was seen in a 25 year old normal female after two first trimester spontaneous abortions. Finally, two index patients, one with a mosaic normal/inv(2)(pl3q11) karyotype (patient 5) and one with a mosaic normal/inv(1) (p13.2p36.2) karyotype (patient 6), were karyotyped because of psychomotor retardation and non-specific dysmorphic stigmata.

\section{Discussion}

Mosaic normal/balanced chromosomal rearrangements are extremely rare events in man, and this is clearly illustrated in the present study. Of the 245 index patients with balanced, reciprocal, autosomal translocations and the 75 index patients with para- and pericentric inversions diagnosed in Leuven in the period 1966 to 1989 , the frequency of mosaic normal/ balanced chromosomal rearrangements was 1 in 81 and 1 in 25 index patients, respectively. In a cytogenetic survey of 334 couples with recurrent spontaneous abortion or fetal wastage, Cantú et al detected a mosaic 46,XY/46,XY,t( $3 ; 4)(\mathrm{q} 22 ; \mathrm{q} 35)$ karyotype in one normal adult male.

The percentage of cells with the balanced chromosomal rearrangement in the present six cases varied between $10 \%$ and $60 \%$, and it was remarkably stable in the two different peripheral blood lymphocyte cultures. This firmly excludes the possibility of in vitro origin of the chromosomal rearrangement. In a recent study of 688 couples with multiple spontaneous abortions, Castle and Bernstein ${ }^{2}$ found mosaic normal/balanced chromosomal rearrangements in nine subjects. However, mosaicism for the chromosomal rearrangement was only found in a small number of cells in one single lymphocyte culture, 


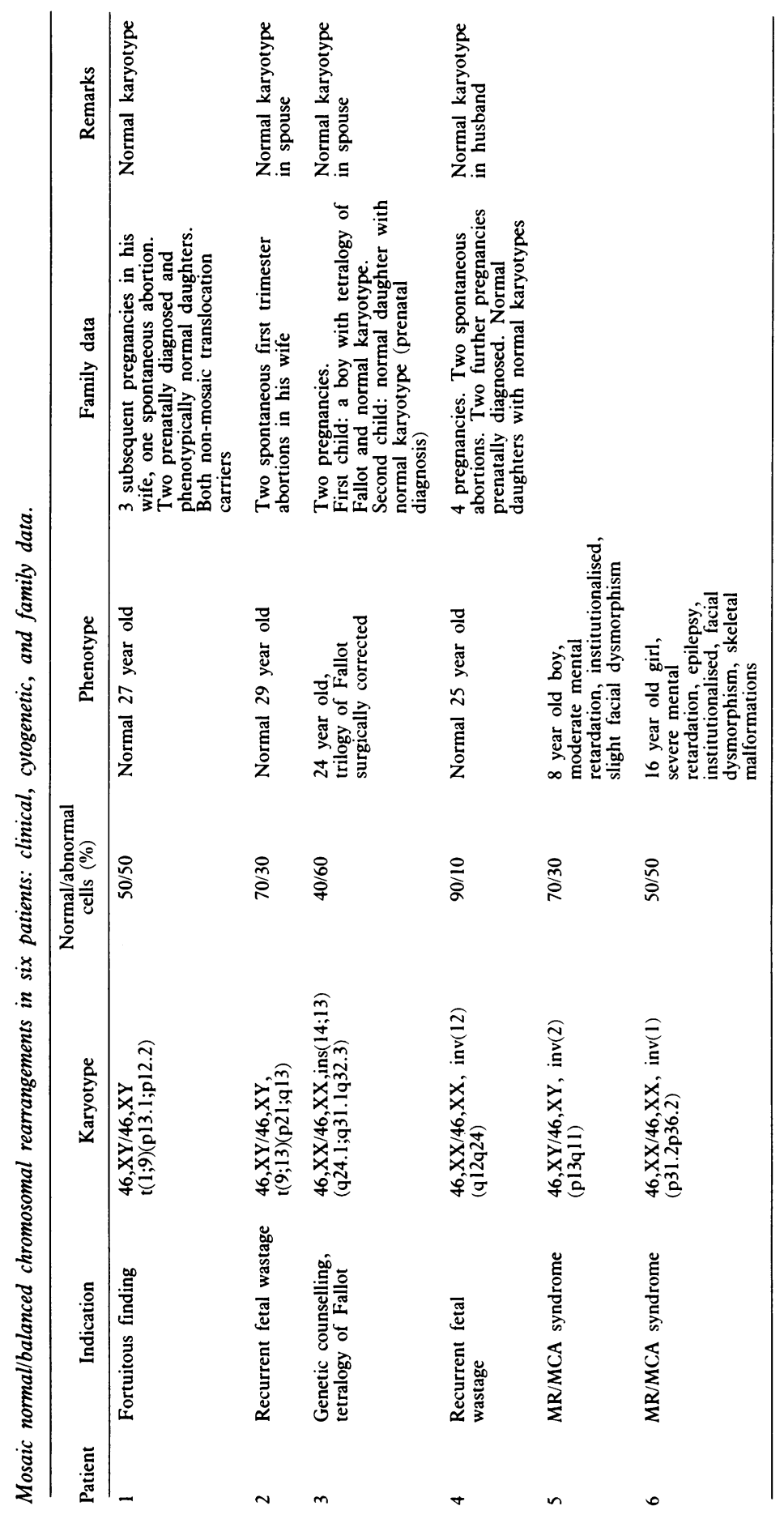


favouring an in vitro origin of the chromosomal changes. On the other hand, Higgins and Palmer ${ }^{3}$ indicated that even a single cell with a balanced translocation among other cells with a normal karyotype might be of pathognomonic significance, particularly in persons referred for a history of multiple spontaneous abortions. The present data clearly illustrate that mosaic chromosomal balanced rearrangements are extremely rare, and they indicate that no diagnostic significance can be given to this type of cytogenetic finding unless it has been confirmed in at least two different peripheral blood lymphocyte cultures or in different tissues.

The most evident and plausible explanation for the origin of mosaic normal/balanced chromosomal rearrangements seems to be a postzygotic event. However, the original hypothesis of Cantú and Ruiz, ${ }^{4}$ based on a prezygotic origin of some structural mosaicism, is tempting as an alternative hypothesis. According to this hypothesis, single strand breaks with subsequent non-homologous hemichromatic joining may occur (before anaphase II) during gamete formation. After fertilisation the replication of such hemitranslocated chromosomes may lead to two cell lines: one normal and the other with a balanced translocation.

Early fetal wastage seems to be the most important consequence in carriers of mosaic normal/balanced chromosomal rearrangements ${ }^{1256}$ (present patients 2 and 4). Another, apparently less frequent, consequence is an MCA/MR syndrome in the carrier ${ }^{7}$ (present patients 5 and 6). An excess of mental retardation and dysmorphic stigmata has been documented in the past in carriers of de novo chromosomal rearrangements in general. The same hypotheses (submicroscopic chromosomal deletion, position effect, etc) to explain the occurrence of an MCA/MR syndrome may be applied when there is mosaicism for a chromosomal rearrangement.

1 Cantú JM, Hernandez A, Jimenez-Sainz M, et al. Aberrationes cromosómicas en 334 individuos con pérdidas gestacionales diversas (incluyendo 144 parejas). Rev Invest Clin 1985;37: $131-4$.

2 Castle D, Bernstein R. Cytogenetic analysis of 688 couples experiencing multiple spontaneous abortions. Am $\mathcal{F}$ Med Genet 1988;29:546-56.

3 Higgins MD, Palmer CG. Single cell translocations in couples with multiple spontaneous abortions. Hum Genet 1987;75:24-7.

4 Cantú JM, Ruiz C. On a prezygotic origin of normal/balanced translocation mosaics. Ann Genet (Paris) 1986;29:221-2.

5 Fryns JP, Kleczkowska A. Reciprocal translocation mosaicism in man. Am J Med Genet 1986;25:175-6.

6 Sciorra L, Ming-Liang L, Cyccurullo G. Translocation mosaicism in a woman having multiple miscarriages. Am $\mathcal{f}$ Med Genet 1986;22:615-7.

7 Saura R, Longy $M$, Serville $F$, Chokairi $O$, Froute MF. Abnormal phenotype in a child with a "balanced" translocation 8/12 in mosaic state. Am $\mathcal{F}$ Med Genet 1987;28:1021-3. 